\title{
A QoS Routing Algorithm for Video Streaming in MANET
}

\author{
Hongbo Huang*
}

Information Engineering Institute, Yueyang Vocational Technical College. Yueyang, Hunan, P.R. China

\begin{abstract}
With the development of wireless communication technology, video communication over the mobile ad hoc networks has been more and more applied. How to ensure the QoS of video communication is an important problem that should be solved quickly. In this paper, we propose a QoS routing algorithm for Video Streaming in MANET. The algorithm introduces multi-constrained QoS routing mechanisms, and optimizes paths through bandwidth, delay, service priorities and congestion degree constraints. The simulation results show that, in medium speed or low-speed MANET where nodes are concentrated, this algorithm can perform well in respects like extending the life of the nodes, reducing packet delay and improving packet delivery fraction.
\end{abstract}

Keywords: Mobile Ad Hoc network, QoS, Multi-path routing, Load balancing, Streaming in MANET.

\section{INTRODUCTION}

With the development of wireless network technology, the video communications based on the mobile ad hoc network (MANET) has found an increasingly wide utilization in many fields such as intelligent transportation, disaster response and military command. In MANET networks, network topology changes frequently, the outage ratio of links is high, energy and transmission distance of a single node are limited, and data transmission needs collaboration between multiple nodes, so routing protocol seems very important $[1$, 2 ]. At the same time, MANET also has problems like narrow channel, strong interference, high error rate, and node mobility. However, video transmission has high demands on the bandwidth, and it is sensitive to delay and packet loss. Therefore, how to ensure the QoS of video communication in multi-hop network is an important problem that should be solved [3].

The present single-path routing protocols such as AODV [4] have the characteristics of easy to manage, configure and realize. But its network transmission efficiency is low, and routing costs a lot, also once the load becomes heavy, it is likely to cause congestion on the local node, and we can not ensure the QoS of video communication. Recently, multipath routing technology has become one of the hottest problems of MANET [5]. AOMDV [6] is a classic on-demand multipath routing protocol. AOMDV chooses two link disjoint paths of which the number of hops is fewest, chooses the way of main and standby routing to communicate, and it improves a lot in the aspect of reducing network overhead and improving network performance compared to AODV. However, AOMDV neglects the change of node flow, so it is difficult for AOMDV to achieve the purpose of load balancing $[7,8]$.

*Address correspondence to this author at the Information Engineering Institute, Yueyang Vocational Technical College. Yueyang, Hunan, 414000, P.R. China; Tel: +86 07308677434; Fax: +86 07308677434 ;

E-mail: 179402061@qq.com
Based on such considerations above, in this paper, we propose a QoS routing algorithm based on MANET network of video communication. This algorithm introduces multiconstrained QoS routing mechanisms, and optimizes paths through bandwidth, delay, service priorities and congestion degree constraints to improve load balancing problems of video communication in MANET.

\section{A QOS ROUTING ALGORITHM FOR VIDEO STREAMING IN MANET}

\subsection{Network Model and QoS Routing Parameter}

Topology of wireless ad hoc networks can be abstracted into a weighted directed graph $\mathrm{G}(\mathrm{V}, \mathrm{E})$, where $\mathrm{V}$ is a set of nodes, and $\mathrm{E}$ is a set of single-hop links. For a viable path whose number of hops is $\mathrm{n} \mathrm{p}=\{\mathrm{v} 1, \mathrm{v} 2, \ldots, \mathrm{vn}\}$, assuming bandwidth, delay, and link congestion degree are its QoS parameter, according to the concave features of QoS parameter, you can define bandwidth and delay as:

$$
\begin{aligned}
& B(p)=\min _{k=1}^{n-1} b\left(v_{k}, v_{k+1}\right) \\
& \operatorname{Delay}(p)=\sum_{k=1}^{n-1} d\left(v_{k}, v_{k+1}\right)
\end{aligned}
$$

Link congestion degree can be defined as:

$$
F^{k}(p)=C(p)^{*} E^{k}(p)
$$

And $F^{k}(p)$ stands for traffic flow congestion degree whose priority is $\mathrm{k}$ in path $\mathrm{p}, \mathrm{C}(\mathrm{p})$ stands for the Buffer ration of service in link $\mathrm{p}$.

We add different service priority queues in the routing buffer list (as shown in Fig. 1) through changing the routing table of AOMDV, and put them in order of priority in buffer strictly. 


Traffic Priority
\begin{tabular}{|c|c|c|c|c|c|c|c|}
\hline 1 & 2 & $\cdots$ & $\mathrm{k}-1$ & $\mathrm{k}$ & $\mathrm{k}+1$ & $\cdots$ & $\mathrm{M}$ \\
\hline S1 & S2 & Sk-1 & $\begin{array}{c}\text { Sk } \\
\text { Occupied buffer }\end{array}$ & $\begin{array}{c}\text { Sk+1 } \\
\text { Om }\end{array}$
\end{tabular}

Fig. (1). Traffic priority queue in the buffer.

For newly arrived priority stream, it can only occupy the buffer whose priority is lower, and there we can get that,

$E^{k}(p)=\sum_{k}^{m} \frac{S_{n}}{S}$

But for all service priorities in feasible path $\mathrm{p}$, there

$$
E^{m}(p) \leq E^{m-1}(p) \leq \ldots \leq E^{k}(p) \leq \ldots \leq E^{1}(p)
$$

The introduction of a priority not only provides priority service of high-class traffics, but can also eases network congestion, and ensures the QoS of service stream better.

\subsection{Implementation of the Optimization of QoS Routing}

Referencing path priority function proposed in the literature [9], considering the constraint of path delay, bandwidth and link congestion degree comprehensively, we can define path preference function $\mathrm{f}(\mathrm{p})$ with multiple QoS constraints,

$f(p)=\alpha \frac{B(p)-B_{\min }}{B_{\text {min }}}+\beta \frac{\text { Delay }_{\text {max }}-\operatorname{Delay}(p)}{\text { Delay }_{\text {max }}}+$

$\gamma($ Congestion $-\mathrm{F}(\mathrm{p}))$

Bigger $f(p)$ means better optimization of path. Here, Bmin and Delaymax stand for the minimum bandwidth and the maximum delay that algorithm specify, Congestion stands for link congestion threshold, $\alpha, \beta$ and $\gamma$ are respectively weighting factors of the three above-described kinds of QoS constraints, and $\alpha+\beta+\gamma=1$.

We add information like Priority_ID, Link min bandwidth, Link congestion degree and Link_max_delay in node route cache through changing the routing table of AOMDV (as shown in Table 1), so we can use $f(p)$ to choose best paths. When a service stream whose priority is $\mathrm{k}$ arrives,

Table 1. The routing table extended.

\begin{tabular}{|c|c|}
\hline Destination & Sequence Number \\
\hline \hline Interface & Advertised Hop Count \\
\hline Last_hop & Next_hop \\
\hline Route_list & Link_Survival Time \\
\hline Route_tag & Link_max_delay \\
\hline Link_min_bandwidth & Link Congestion Degree \\
\hline Priority_ID & Congestion Threshold \\
\hline
\end{tabular}

the route discovery mechanism of AOMDV will be started first, source nodes will initiate route request RREQ, and RREQ is to be broadcast to the neighbor nodes. If any intermediate node receives multiple RREQ, it will record reverse route that 2 RREQ packet with biggest $f(p)$ pass. Then target nodes will send route reply (RREP) to source nodes, and intermediate nodes will feedback these paths recorded to source nodes through the shortest path that can be found. The source nodes build their own network topology diagrams by superimposing these paths information, and then choose 2 possible paths whose bandwidth, delay and congestion degree are best, from these diagrams.

\subsection{Maintenance and Update of Routing}

Choosing the best two routes as the primary and the alternate path to send service stream can avoid nodes with great congestion degree, also can distribute traffic better, and it is good for the optimization of network resources. In the process of the data transmission, every link node maintains connectivity between adjacent nodes by sending Hello packet (RREP without request), and keeping the latest routing information according to survival time and serial numbers. Each node has its own network topology diagram, no matter which node that data goes through, and these nodes can find the appropriate path from their topology diagrams to send data.

When the primary route reaching the destination node fails, the destination node broadcasts route error information (RERR) to the source node and each intermediate node. When the source node sends the next packet, it will choose an alternate route as the primary route to send data, at the same time, the source node will find a new route to replace failure path from network topology diagrams. Only when the two paths fail, the source node will launch a new route to request a new search path. So, it inherits the advantages of multi-path routing of AOMDV well, and can maintain the stability and adaptability of path better, reduce control overhead caused by frequent failure of routing.

\section{SIMULATION ENVIRONMENT AND RESULTS}

This experiment used the Foreman video sequence, a software named JM14 based on JVT/H.264 video encoding compression standard to encode it. The sequence format is CIF $(352 \times 288)$, and the sending speed is $30 \mathrm{fps}$. We created a network topology with 50 nodes in $800 \mathrm{~m} \times 600 \mathrm{~m}$ space, and selected two nodes randomly from the 50 nodes as the sender and the recipient. The simulation parameters are shown in Table 2. Video data uses UDP protocol to transmit in wireless multi-hop network, and each frame of video se- 
Table 2. Simulation parameters.

\begin{tabular}{|c|c|c|c|}
\hline Name & Parameters & Name & Parameters \\
\hline \hline Space Size & $800 * 600$ & Node Speed(m/s) & $30,18]$ \\
\hline Number of Nodes & 50 & Pause Time & $900 \mathrm{~s}$ \\
\hline Movement Model & Random Waypoint & Link Delay & {$[20,50] \mathrm{ms}$} \\
\hline Data Packet Size & $1024 \mathrm{~B} / 512 \mathrm{~B}$ & Delay constraint & $800 \mathrm{~ms}$ \\
\hline MAC & 802.11 & Bandwidth constraint & $256 \mathrm{~Kb} / \mathrm{s}$ \\
\hline RF Transmission Range & $250 \mathrm{~m}$ & & \\
\hline
\end{tabular}

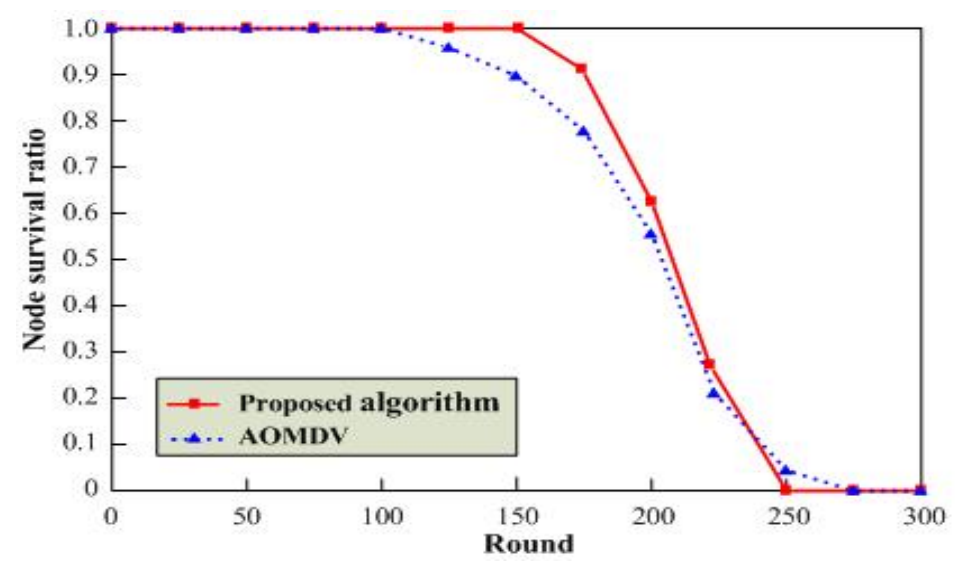

Fig. (2). The lifetime of the network with varying loads.

quence is encapsulated into plural UDP packets, and each data packet length is not greater than 1024 bytes. Extract parity frames from the video coding sequence to implement multi-path transmission. Set the receiver play buffer size to $5 \mathrm{~s}$ for smoothing the packet delay jitter to improve the play fluency. Simulation time is 900 seconds. The result of the simulation performance takes the average of results of 5 simulations.

\subsection{Network Lifetime Test}

The network lifetime reflects the survival rates of network nodes and the stability of the whole network to a certain extent. Death of link nodes is generally due to local congestion and energy depletion. Therefore, we test our algorithm and ability to balance load of AOMDV algorithm by increasing the amount of data sent in a static network environment. Other nodes keep sending CBR packets at a speed of $512 \mathrm{~B}$ per second as source node sends video stream to target node. We can see it from Fig. (2) that, with the amount of data sent increasing, network load will be heavier, and dead nodes will be gradually increased. Because the proposed algorithm uses multi-constrained QoS routing, it can distribute traffic according to the degree of nodes congestion. At the time when network appears dead node obviously lags AOMDV, and in the same rounds which have many nodes, more nodes survive. But with survival, nodes of the whole network become fewer and fewer, which causes that feasible paths become scarce, and proposed algorithm is not as good as AOMDV algorithm because of the additional routing overhead due to multi-constrained compute. This shows proposed algorithm is more suitable for network environment with concentrated nodes.

\subsection{Packet Delivery Fraction and End-to-End Delay}

We created multiple sports scene by setting different node speed to test the network throughput performance of AOMDV protocol and the proposed algorithm under different sports scene. In video communication, delay and packet delivery ratio are two important performance indicators that affect video communication quality. From Fig. (3) we can see that, in MANET where speed of nodes is less than $14 \mathrm{~m} / \mathrm{s}$, average packet delay of proposed algorithm is lower than AOMDV. At the same time, packets decoded in the destination node are also more (as shown in Fig. 4). This is because in the network topology of the low and medium speed, the network is stable, ideal accessible paths are abundant, and the link is reliable. Because the proposed algorithms uses multi-constrained optimal path and maintains a path through the network topology, it can exhibit better 


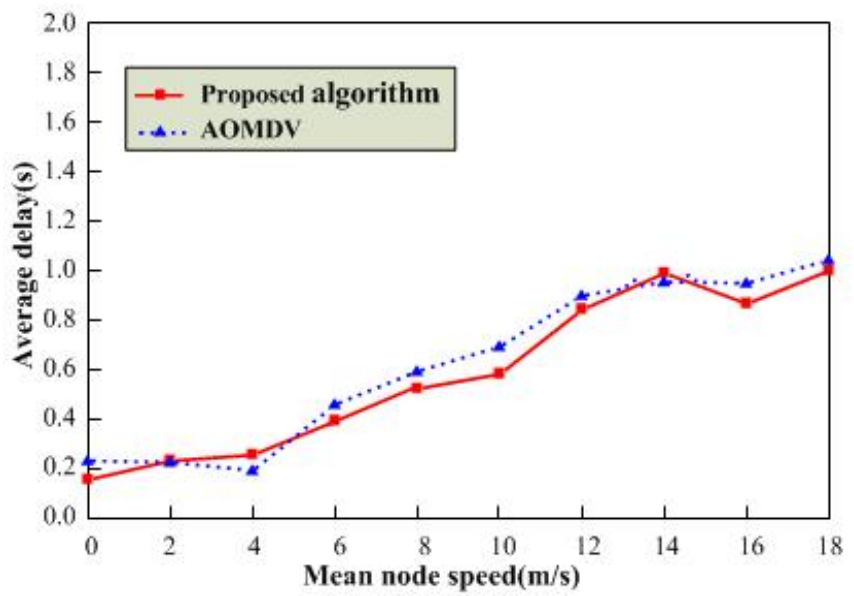

Fig. (3). Average packet delay with varying mobility.

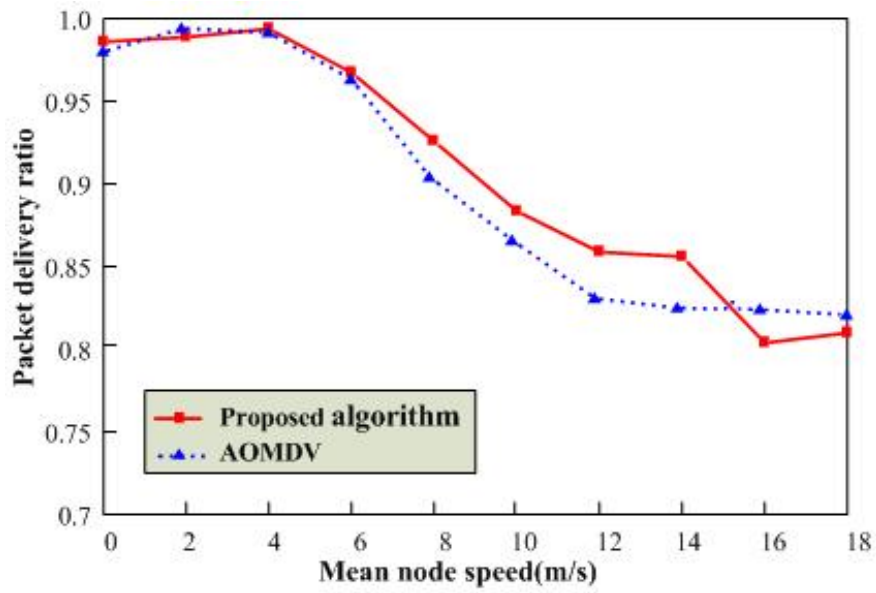

Fig. (4). Packet delivery ratio with varying mobility.

fault tolerance and stability. But in high speed environment, it cannot perform well because active path fails frequently, and the probability of packet retransmission failure increases.

\section{CONCLUSION}

In this paper, we propose a QoS routing algorithm for video streaming in MANET aiming at issues of traffic balancing and quality of service of multi-path video transmission over MANET. The algorithm introduces multiconstrained QoS routing mechanisms in AOMDV, and optimizes paths through bandwidth, delay, service priorities and congestion degree constraints. The simulation result shows that, in medium-speed or low-speed MANET where nodes are concentrated, the proposed algorithm performs well in aspects like extending the life of the nodes and improving network throughput.

\section{CONFLICT OF INTEREST}

The author confirms that this article content has no conflict of interest.

\section{ACKNOWLEDGEMENTS}

This work was financially supported by the Hunan Province Science and Technology Project Foundation of China (Grant No. 2014FJ6087).

\section{REFERENCES}

[1] S. Mueller and D. Ghosal, Multipath Routing in Mobile Ad Hoc Networks: Issues and Challenges, Lecture Notes in Computer Science, Springer, Berlin, pp. 209-234, 2004.

[2] M.I. Abdullah, M.M. Rahman, Ahsan-ul-Ambia, and M.Z. Mahmud, "Performance of conferencing over MANET routing protocols", ARPN Journal of Systems and Software. vol. 2, no. 6, pp. 214-218, 2012.

[3] X.G. Zhang, and Z.M. Guo, "Rate-distortion optimized multi-path selection for video streaming over wireless multi-hop networks", Journal of Software, vol. 22, no. 10, pp. 2412-2424, 2011.

[4] C.E. Perkins, E.M. Belding-Royer, and I. Chakeres, Ad hoc on demand distance vector (AODV) Routing, IETF Internet Draft, draft-perkins-manet-aodvbis-00.txt, 2003.

[5] H. An, and X. Lu, "A review of routing protocols for mobile Ad Hoc networks", Computer Engineering and Science. vol. 28, no. 2, pp. 4-9, 2006.

[6] K.M. Mahesh, and R.D. Samir, "Ad hoc on-demand multipath distance vector routing", Wireless Communications and Mobile Computing, vol. 6, pp. 969-988, 2006. 
[7] P. Hu, and J. Zhang, "Improved AOMDV routing protocol based on energy-balanced strategy", Computer Engineering and Design, vol. 32, no. 9, pp. 2976-2979, 2011.

[8] J. Yang, Z. Fan, and X. Wan, "Design of QoS based AOMDV routing protocol in Ad hoc network", Computer Engineering and Application, vol. 47, no. 7, pp. 85-88,2011.
[9] X. Cao, R. Wang, H. Huang, L.J. Sun, and F. Xiao, "Multi-path routing algorithm for video stream in wireless multimedia sensor networks", Journal of Software, vol. 23, no. 1, pp. 108-121, 2012.

Received: September 16, 2014

Revised: December 23, 2014

Accepted: December 31, 2014

(C) Hongbo Huang; Licensee Bentham Open.

This is an open access article licensed under the terms of the Creative Commons Attribution Non-Commercial License (http://creativecommons.org/licenses/by$\mathrm{nc} / 4.0 /$ ) which permits unrestricted, non-commercial use, distribution and reproduction in any medium, provided the work is properly cited. 